\title{
ROBUST DIGITAL WATERMARKING TECHNIQUES BASED ON DCT AND DWT ALGORITHMS
}

\author{
Mohammed A. Al- Jarrah \\ Department of Computer Engineering \\ Yarmouk University \\ Irbid, Jordan
}

\author{
Mariam Manasrah \\ Department of Computer Engineering \\ Yarmouk University \\ Irbid, Jordan
}

\begin{abstract}
- copyright protection of digital media has become a major concern for owners of digital media such as images, videos, and audios. Digital watermarking is a process of hiding an image or text on an image to provide evidence of its authenticity. In this paper, we developed five different methods. We considered the transform of an image using DCT, DWT and DCT with DWT formats could affect the hidden watermark in a way or another. In addition, we considered adding additional attacks like crop image, salt and pepper noise, Gaussian noise and filter. The schemes we are providing here are resilient to these types of attacks and is novel in this scenario.
\end{abstract}

Keywords-Digital image watermarking, image copyright protection, frequency domain watermarking, discrete wavelet transform (DWT), and discrete cosine transform (DCT).

\section{INTRODUCTION}

Digital image watermarking has been proposed to prevent illegal and malicious copying and distribution of digital images. The procedure can be simply achieved by embedding unnoticeable information called a watermark into the image content. The watermark could be a random number sequence, copyright messages, or ownership identifier $[1,2]$. Digital watermarking has a wide range of practical applications such as digital cameras, digital libraries, medical imaging, content identification and management, document and image security, and many others [3].

Effective watermarking has many requirements, the most important of which are imperceptibility (perceptual transparency) and robustness. Imperceptibility requires the watermarking algorithm to embed the watermark information in the host image in such a way that the quality of the underlying host image is not affected [4].

In this paper, we propose a blind watermark, imperceptible and robust watermarking technique. We considered the transform of an image using Discrete Cosine Transform (DCT), Discrete Wavelet Transform (DWT) and DCT with DWT [5, 6], that could affect the hidden watermark. Also, we considered adding additional attacks like crop image, salt and pepper noise, Gaussian noise and filter. The schemes we are providing here are resilient to these types of attacks and is novel in this scenario.

Our contribution could be summarized in few points: first we are introducing a blind watermarking scheme. In which we did require the cover image to recover the watermark. Second, we are proposing different attacks. Third we are proposing and comparing five different algorithms, which are, DWT using pseudo random noise, DCT using pseudo random noise, DWT using comparison between mid-band coefficients, DCT using comparison between mid-band coefficients and fifth combines both algorithms. We compared all five algorithms and commented on their performances. In the next section, a brief introduction to DWT and DCT and their relevance to digital watermarking is introduced. In section three, we proposed DWT algorithm using pseudo random noise. Watermarking image based on DWT using comparison between mid-band coefficients. In section four. Section five explain DCT algorithm using pseudo random noise. DCT algorithm based on comparison between mid-band coefficients and DWT join with DCT algorithm in section six and seven respectively. Result and performance evaluation in section eight and finally conclusion in section nine.

\section{DCT AND DWT BASED WATERMARKING}

In this section, we provide brief description of the DCT and DWT techniques, and show how they are applied in digital watermarking.

\subsection{DWT Based Watermarking}

For digital images, applying DWT corresponds to processing the image by 2 -D filters. The filters divide the input image into four non-overlapping sub-bands LL, LH, HL and HH. The LL sub-band represents Approximation coefficients while the LH, HL and HH sub-bands represent the Horizontal, Diagonal and Vertical coefficients respectively. To obtain the next level of wavelet coefficients, the LL sub-band is further processed until some final scale $\mathrm{N}$ is reached. When $\mathrm{N}$ is reached, we will have $3 \mathrm{~N}+1$ sub-bands consisting of the multiresolution sub-bands LLN and LHx, HLx and HHx where $\mathrm{x}$ ranges from 1 to $\mathrm{N}$ [7]. 


\subsection{DCT based watermarking}

Discrete Cosine Transform (DCT) is a popular transform domain watermarking technique. It is allow an image to be broken up into three different frequency bands the high, middle and low frequency, thus making it easier to choose the band in which the watermark is to be inserted. Generally, the middle frequency bands are chosen because embedding the watermark in middle frequency band does not lose the watermark information to most visual important parts of the image [8].

\subsection{Proposed hybrid DWT-DCT based watermarking}

The DWT and DCT are different transform domain techniques and thus provide different levels of robustness against the same attack.

\section{IMAGE WATERMARKING BASED DWT USING PSEUDO RANDOM NOISE}

Most of the image energy is concentrated at the lower frequency sub-band LL. Therefore, embedding watermarks in the lower sub-band may degrade the image significantly. Embedding in the low frequency sub-bands, however, could increase robustness significantly. On the other hand, the high frequency sub- band $\mathrm{HH}$ include the edges and textures of the image, for which the human eye is generally sensitive to changes in such sub-band. The compromise adopted by many DWT-based watermarking algorithm, is to embed the watermark in the middle frequency sub-bands LH and HL where acceptable performance of imperceptibility and robustness could be achieved [9].

Applying decoding procedure by using provided key code to generate a pseudo random noise (PRN) matrix then perform a 2D single level wavelet transform to obtain the four coefficients matrices (A, H, D, V) corresponding to approximation, horizontal, diagonal and vertical coefficients sub-bands respectively. Update the V \& D coefficients according to the formulas:

$$
\begin{aligned}
& \mathrm{V}^{\prime}=\mathrm{V}+\mathrm{K} * \mathrm{PRN} \\
& \mathrm{D}^{\prime}=\mathrm{D}+\mathrm{K} * \mathrm{PRN}
\end{aligned}
$$

The formulas are applied based on the watermark value. If the $\mathrm{W}$ (i) $=0$ then the coefficients are updated, otherwise nothing changed. $\mathrm{W}$ is the watermark reshaped into vector and the index $i$ represent the index of the pixels moving with the block index. After the coefficients were modified, apply the inverse wavelet transform using the new values. This will produce a watermarked image. Continue moving over all watermark pixels and image blocks. When done accumulate the image back into original format.

In decoding procedure perform a $2 \mathrm{D}$ single level wavelet transform for watermarked image to obtain the four coefficients matrices (Aw, Hw, Dw, Vw). Generate pseudo random noise sequence (PRN) using the same seed used in the watermark embedding algorithm and then run the correlation function between each block (Vw, PRN) once and (Dw, PRN) once. Compare the value of the correlation to a certain threshold and set or reset a watermark vector based on this comparison. Repeat for the watermark length.

\section{IMAGE WATERMARKING BASED DWT USING COMPARISON BETWEEN MID-BAND COEFFICIENTS}

For decoding procedure performed by applying a 2D single level wavelet transform to obtain the four coefficients matrices (A, H, D, V), now pick the V coefficients. The new watermark (NW) size from size of the $\mathrm{V}$ coefficient matrix divided by block size (8X8). Now from the beginning of watermark (W) until length of the original watermark pad it by the original watermark values and the others equal one. Depends on the size of the NW we do this procedure: transform each block in $\mathrm{V}$ matrix using 2D single level wavelet transform. If the NW (i) $=0$ then if (3) formula achieved then do swap between this pixels, otherwise no changes.

$$
\text { IF (CA_bV (u1, v1) < CA_bV (u2, v2)) }
$$

But, if NW (i) $=1$ then if formula (4) achieved then do swap between this pixels, otherwise no changes.

$$
\text { IF (CA_bV (u1, v1) < CA_bV (u2, v2)) }
$$

Apply this procedure for all other coefficients $\left(\mathrm{CH}_{-} \mathrm{bV}\right.$, CD_bV, CV_bV). After the coefficients were modified, apply the inverse wavelet transform twice using the new values. This will produce a watermarked image. Continue moving over all watermark pixels and image blocks. When done accumulate the image back into original format using the new values.

In decoding procedure perform a $2 \mathrm{D}$ single level wavelet transform for both original image and watermarked image to obtain the four coefficients matrices for original image (Ao, Ho, Do, Vo) and other four coefficients for watermarked image ( $\mathrm{Aw}, \mathrm{Hw}, \mathrm{Dw}, \mathrm{Vw}$ ) corresponding to approximation, horizontal, diagonal and vertical coefficients sub-bands respectively. Then perform a 2D single level wavelet transform for Vo block and $\mathrm{Vw}$ block to obtain another four coefficients matrices for Vo(CA_bVo, CH_bVo, CD_bVo, CV_bVo) and four coefficient matrices for $\mathrm{Vw}$ (CA_bVw, CH_bVw, CD_bVw, CV_bVw). Now run the correlation function between blocks (CA_bVo, CA_bVw), (CH_bVo, CA_bVw), (CD_bVo, CD_bVw) and (CV_bVo, CV_bVw). Sum the values of the correlations and multiply the result by key $(\mathrm{K})$, the value for key depends on the clarity of extracted watermark image. Compare the final result of the correlations to a certain threshold and set or reset a watermark vector based on this comparison. Repeat for the watermark length. Reshape the watermark matrix into the original watermark size. Compare the reconstructed watermark with the original watermark.

\section{IMAGE WATERMARKING BASED ON DCT TRANSFORM USING PSEUDO RANDOM NOISE}

For encoding procedure performed by applying a 2D DCT transform. Using the provided key code generate pseudo 
random noise (PRN) matrix. Now pick the coefficients and update them according to the formulas:

$$
\mathrm{T}^{\prime}=\mathrm{T}+\mathrm{K} * \mathrm{PRN}
$$

The formulas are applied based on the watermark value. If the $\mathrm{W}$ (i) $=0$ then the coefficients are updated, otherwise nothing changed. $\mathrm{W}$ is the watermark reshaped into vector and the index $i$ represent the index of the pixels moving with the block index. After the coefficients were modified, apply the inverse cosine transform using the new values. This will produce a watermarked image. Continue moving over all watermark pixels and image blocks.

The decoding procedure performed by applying a 2D DCT transform onto watermarked image (WI).

Run the correlation function between (WI, PRN) blocks. Then compare the value of the correlation to a certain threshold and set or reset a watermark vector based on this comparison. Repeat for the watermark length.

\section{IMAGE WATERMARKING BASED ON DCT USING} COMPARISON BETWEEN MID-BAND COEFFICIENTS

The encoding procedure performed by creating new watermark (NW) size equal to the size of cover image divided by block size (8X8). Now from the beginning of NW until size of the original watermark padding it by the original watermark values and the others equal one. Depends on the size of the NW we do this procedure:

a) Transform each block in cover image matrix using DCT transform.

b) If the NW (i) $=0$ then if formula (6) achieved then do swap between this pixels, otherwise no changes.

IF (dct_block (u1, v1) < det_block (u2, v2))

Then we adjust the two values such that their difference $>=\mathrm{k}$.

c) But if NW (i) $=1$ then if (7) formula achieved then do swap between this pixels, otherwise no changes.

IF (dct_block (u1, v1) < det_block (u2, v2))

Now apply the inverse of DCT using the new values and continue moving over all image blocks, this will produce a watermarked image. When done accumulate the watermarked image back into original format.

Decoding procedure performed by applying a 2D DCT transform for each block perform DCT transform and then depending on the comparison result between values of DCT block, we retrieve the watermark values. Repeat for the watermark length, which could run on all image blocks.

\section{IMAGE WATERMARKING USING DWT WITH DCT}

In order to achieve the advantages of both DWT and DCT transforms, we decided to combine both of them in a single watermarking algorithm. In this section we consider the application of the both DWT and DCT to enhance the algorithms described in previous sections.

In encoding procedure we will start with the wavelet based procedure and end with the cosine transform based procedure Thus, perform DWT transform for each block in cover image to obtain the four coefficients matrices (A, H, D, V), pick V and perform DCT transform to obtain the coefficients matrix. Now apply formula based on the watermark value, if $\mathrm{W}$ (i) $=0$, the coefficients are updated, otherwise nothing changed. Then we adjust the two values such that their difference $>=k$. Also if $\mathrm{W}$ (i) $=0$ then apply this formula using pseudo random noise (PRN)

$$
\text { Dct_cv_block=Dct_cv_block+K*PRN }
$$

where, $\mathrm{W}$ is the watermark reshaped into vector and the index $\mathrm{i}$ represent the index of the pixels moving with the block index. Apply inverse DCT into dct_cv_block. After the V coefficients were modified, Perform inverse DWT. Continue moving over all watermark pixels and image blocks. When done accumulate the watermarked image back into original format.

In decoding procedure we need the watermarked image and a PRN then for each block in watermarked image to perform a DWT transform to obtain the four coefficients matrices ( $\mathrm{Aw}, \mathrm{Hw}, \mathrm{Dw}, \mathrm{Vw}$ ). Run the correlation function between each block (Vw, PNR). Compare the value of the correlation to a certain threshold and set or reset a watermark vector based on this comparison. For each vertical block $\mathrm{Vw}$ perform a DCT transform to obtain the coefficients matrix. Now depending on the result from comparing two pixels in vertical block we make changes on watermark vector, to recover the original values.

Repeat for the watermark length, which could run on all image blocks. After that reshape the watermark matrix into the original watermark size.

\section{RESULTS AND PERFORMANCE EVALUATION}

We evaluated the performance of our proposed image watermarking algorithm using a 512 × 512 grayscale images, 'Lena', 'Mandrill' and 'cameraman'. Block size for all five algorithms is $8 \times 8$. The watermark is $20 \mathrm{X} 50$ pixels 'Copyright' binary image. The key with different values depends on method.

To evaluate of the proposed algorithms we use the Structural Similarity (SSIM) [10], Correlation (CORR) and Peak Signal to Noise Ratio (PSNR) to evaluate the watermarked image transparency, and the Mean Absolute Error (MAE) [10] to evaluate the extracted watermark correctness.

To verify the robustness of the proposed watermarking schemes. Four types of attacks were added; crop watermarked image, second add salt and pepper noise, third add Gaussian 
noise, fourth attacked watermarked image using Low pass filter [10].

We tuned the gain factor, which denoted by $\mathrm{K}$ in the equations (1), (2) and (5), from 0.1 to 2 with an increment of 0.05 . The gain factor is a measure of the strength of the watermark embedding and it is directly reflecting the robustness of the procedure. Hence, we need to find a value, which could retain the watermark with the least possible disturbance of the cover image.

Due to space limitations, experimental results for extracted watermark based on DCT using comparison between mid-band coefficients are only presented. Robustness of the algorithms was measured by comparing the original watermark with the extracted watermark using the structural similarity, correlation and peak signal to noise ratio.

Figure 1 shows the original three images, Lena, Mandrill and cameraman. The results for watermarked images are shown in Figure 2. In Figure 3, we dipects the extracted watermark after applying four attacks using DCT algorithm based on comparison between mid-band coefficients with results for Mean Absolut Error (MAE). Referring to the results in Table 1, the first column shows five algorithms and second column for Lena's robustness results, third column for Mandrill and forth for Cameraman results. PSNR for DWT using comparison between mid-band coefficients equal 49.7944, 48.6765, 48.4553 for Lena, Mandrill and Cameraman respectively, which mean that cover image almost not affected using embedding watermark. In addition, those reflect on correlation and similarity, which have higher values nearly to one.
The worst one is DCT using pseudo random noise generator with SIMM equal .44497, .675329, .434274 for Lena, Mandrill and Cameraman respectively. Second DWT joined with DCT, third DCT using comparison between midband coefficients, forth DWT using pseudo random noise. However, after analyses attack tables depends on MAE we conclude that the best algorithm to survive watermark after attacks is almost DCT-mid band then DWT-DCT join.

Wavelet and cosine transforms are providing an excellent robustness level for the watermarked image. Watermarked images are matching the original with $80 \%$ and above. All algorithms show close results for CORR. DWT using comparison between coefficients requires different values of the factor to extract the watermark.

\section{CONCLUSION}

Researching in the field of digital watermarking has been steadily gaining momentum. For different media types including images, audio and video, many new methods have been published. In this paper, we proposed new digital image watermarking algorithms. The proposed algorithms make use of two powerful mathematical transforms; DWT and DCT. In addition, these techniques were combined to exploit their respective attractive features. Simulation results explained the effectiveness of our proposed method with regard to the essential watermarking requirements including robustness, and imperceptibility.

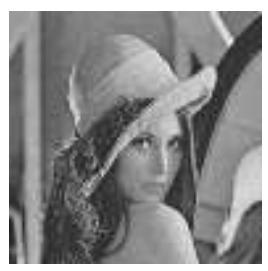

Original Lena

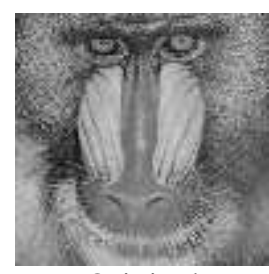

Original Mandrill

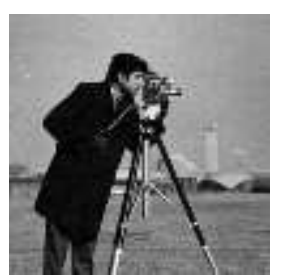

Original

Cameraman

Figure 1. Original images.

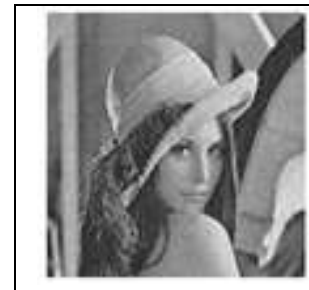

DWT using pseudo random noise

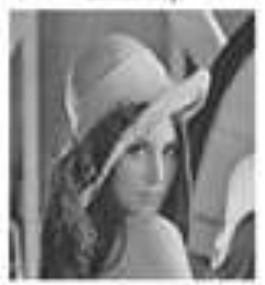

DWT using

comparison between mid-band coefficients

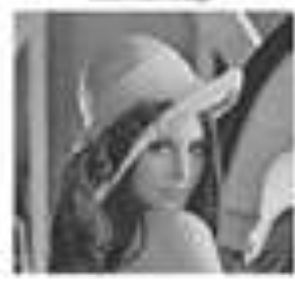

DCT using comparison between mid-band coefficients

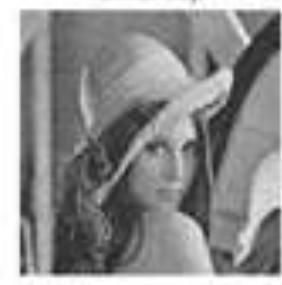

DCT using pseudo random noise

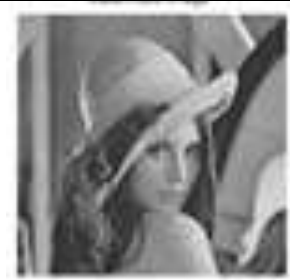

DWT with DCT

Figure 2. Watermarked 'Lena' using five different algorithms 
Proc. of The Sixth Intl. Conf. On Advances In Computing, Control And Networking - ACCN 2017 Copyright (C) Institute of Research Engineers and Doctors, USA .All rights reserved.

ISBN: 978-1-63248-117-7 doi: 10.15224/ 978-1-63248-117-7-09

\begin{tabular}{|c|c|c|c|c|}
\hline Original watermark & Cropping & Salt and pepper & Gaussian noise & Low pass filter \\
\hline \multirow{2}{*}{ Ergpright } & L'opyright & Copyright & Espyright. & Copyright \\
\hline & $\begin{array}{l}\text { Block size }: 8 \times 8 \\
\text { K : } 50 \\
\text { MAE }: 0.038\end{array}$ & $\begin{array}{l}\text { Block size : } 8 \times 8 \\
\text { K }: 50 \\
\text { MAE }: 0.016\end{array}$ & $\begin{array}{l}\text { Block size : } 8 \times 8 \\
\text { K : } 50 \\
\text { MAE : } 0.013\end{array}$ & $\begin{array}{l}\text { Block size }: 8 x 8 \\
\text { K : } 50 \\
\text { MAE }: 0.00001\end{array}$ \\
\hline
\end{tabular}

Figure 3. Extracted watermark using DCT based on comparison between mid-band coefficients

Table 1. Robustness results for proposed algorithms

\begin{tabular}{|l|l|l|l|l|l|l|l|l|l|}
\hline & \multicolumn{3}{|c|}{ Lena } & \multicolumn{3}{c|}{ Mandrill } & \multicolumn{3}{c|}{ Cameraman } \\
\hline $\begin{array}{l}\text { DWT using pseudo } \\
\text { random noise }\end{array}$ & PSNR & CORR & SSIM & PSNR & CORR & SSIM & PSNR & CORR & SSIM \\
\hline $\begin{array}{l}\text { DWT using } \\
\text { comparison } \\
\text { between mid-band } \\
\text { coefficients }\end{array}$ & 37.76 & 0.9775 & 0.585712 & 36.9786 & 0.968653 & 0.785577 & 38.1075 & 0.987018 & 0.563942 \\
\hline $\begin{array}{l}\text { DCT using } \\
\text { comparison } \\
\text { between mid-band } \\
\text { coefficients }\end{array}$ & 49.79 & 0.9999 & 0.99922 & 48.6765 & 0.999849 & 0.999254 & 48.4553 & .999888 & 0.999116 \\
\hline $\begin{array}{l}\text { DCT using } \\
\text { pseudo random } \\
\text { noise }\end{array}$ & 40.83 & 0.9938 & 0.839408 & 38.6882 & 0.985188 & 0.901719 & 41.0474 & 0.996619 & 0.820534 \\
\hline DCT with DWT & 36.25 & 0.9525 & 0.44497 & 35.472 & 0.940096 & 0.675329 & 36.642 & 0.974769 & 0.434274 \\
\hline $\begin{array}{l}\text { DWT using pseudo } \\
\text { random noise }\end{array}$ & 43.96 & 0.9985 & 0.95314 & 42.124 & 0.996933 & 0.989645 & 44.5197 & 0.999314 & 0.945846 \\
\hline
\end{tabular}

\section{REFERANCES}

[1] M. Khan, M. Rahman and I. Saker, "Digital Watermarking for Image Authentication Based on Combined DCT, DWT and SVD Transformation". International Journal of Computer Science Issues, Volume 10, Issue 3, No. 1, May 2013.

[2] M. Tech, "Transform Based Digital Image Watermarking Techniques for Image Authentication", International Journal of Emerging Technology and Advanced Engineering, Volume 4, Issue 5, May 2014.

[3] C. Chang, T. Nguyen and C. Lin,"A Blind Reversible Robust Watermarking Scheme for Relational Databases", Scientific World Journal, 2013.

[4] H. Ranotkar, M. Deshmukh, "Privacy Enhancement of Data with Safe Watermark Extraction Using Signal Processing", International Journal of Application or Innovation in Engineering and Management, Vol. 3, Issue 11, November 2014.

[5] S. Singh, P. Rawat and S. Agrewal, "A Robust Watermarking Approach using DCT-DWT", International
Journal of Emerging Technology and Advanced Engineering, Volume 2, Issue 8, August 2012.

[6] S. Indrabi and Sheenam, "Watermarking Digital Images: A Hybrid Approach", International Journal of Advanced Research in Computer Science and Software Engineering, Volume 5, Issue 5, May2015.

[7] T. Modi, P. Anilkumar and J. Alex, "Low Complexity DWT Architecture Implementation for Feature Extraction using Different Multipliers", Indian Journal of Science and Technology, Volume 8, Issue 21, September 2015.

[8] S. Singh, A. Rawat, and S. Agrawal, "A Literature Review on Water Marking Techniques," International Journal of Scientific Engineering and Technology, Volume No.1, Issue No.4, pp: 21-23. 2012.

[9] R. Philip and M. Sumithra, "SVD Based Watermarking Method for Medical Images Security”, International Journal of Computer Applications, Volume 66-No. 2, March 2013.

[10] C. Chen, Y. Tang and W. Hsieh,'Print-and Scan Resilient Watermarking through Polarizing DCT COEFFICIENTS", IEICE Transaction on Information and Systems, Volume E96-D, No.10, October 2013. 